\title{
Psychological interventions for improving adherence to inhaled therapies in people with cystic fibrosis (Protocol)
}

Dawson S, Cowap L, Clark-Carter D, Girling CJ

Dawson S, Cowap L, Clark-Carter D, Girling C-J.

Psychological interventions for improving adherence to inhaled therapies in people with cystic fibrosis (Protocol).

Cochrane Database of Systematic Reviews 2020, Issue 10. Art. No.: CD013766.

DOI: 10.1002/14651858.CD013766.

www.cochranelibrary.com

Psychological interventions for improving adherence to inhaled therapies in people with cystic fibrosis (Protocol) 
TABLE OF CONTENTS

HEADER 1

ABSTRACT

BACKGROUND

OBJECTIVES

METHODS

ACKNOWLEDGEMENTS

REFERENCES

APPENDICES

HISTORY

CONTRIBUTIONS OF AUTHORS

DECLARATIONS OF INTEREST

SOURCES OF SUPPORT 
[Intervention Protocol]

\section{Psychological interventions for improving adherence to inhaled therapies in people with cystic fibrosis}

Sophie Dawson 1,2 , Lisa Cowap², David Clark-Carter², Carla-Jane Girling ${ }^{3}$

1Wolfson Cystic Fibrosis Centre, Nottingham University Hospitals NHS Trust, Nottingham, UK. 2Staffordshire Centre for Psychological Research, School of Life Sciences \& Education, Staffordshire University, Stoke-on-Trent, UK. ${ }^{3}$ Sheffield Clinical Trials Research Unit, ScHARR, University of Sheffield, Innovation Centre, Sheffield, UK

Contact address: Sophie Dawson, Sophie.Dawson@nuh.nhs.uk.

Editorial group: Cochrane Cystic Fibrosis and Genetic Disorders Group.

Publication status and date: New, published in Issue 10, 2020.

Citation: Dawson S, Cowap L, Clark-Carter D, Girling C-J. Psychological interventions for improving adherence to inhaled therapies in people with cystic fibrosis (Protocol). Cochrane Database of Systematic Reviews 2020, Issue 10. Art. No.: CD013766. DOI: 10.1002/14651858.CD013766.

Copyright @ 2020 The Cochrane Collaboration. Published by John Wiley \& Sons, Ltd.

\section{A B S T R A C T}

\section{Objectives}

This is a protocol for a Cochrane Review (intervention). The objectives are as follows:

The primary objective of the review is to assess the efficacy of psychological interventions for improving adherence to inhaled therapies in people with cystic fibrosis (CF). A secondary objective is to establish the most effective components, or behaviour change techniques (BCTs), of interventions for improving adherence to inhaled therapies in people with CF, using the BCT Taxonomy version 1 (Michie 2013). 


\section{B A C K G R O U N D}

\section{Description of the condition}

Cystic fibrosis (CF) is a chronic, genetic condition affecting over 10,500 people in the UK (UK CF Registry 2019), 30,000 people in the USA (CF Foundation Patient Registry 2019), and around 90,000 people worldwide (Bell 2020). One in 25 people in the UK carry the faulty gene that causes CF, usually without realising it (Cystic Fibrosis Trust 2018). People with CF are prone to recurrent chest infections, or pulmonary exacerbations, due to the build-up of thick, sticky mucus, or sputum, in their lungs and digestive systems.

Although people with CF are living longer, their life expectancy remains well below average, and the median predicted survival age of someone born with CF in 2018 ranges between 47.3 years in the UK (UK CF Registry 2018), 47.4 years in the USA (CF Foundation Patient Registry 2019), and 52.1 years in Canada (Cystic Fibrosis Canada 2019). As lung disease is the primary cause of morbidity and mortality in people with CF, nebulised medications, or inhaled therapies, are usually prescribed (ECFS Patient Registry 2017), with multiple trials demonstrating their efficacy (Smith 2018; Yang 2018). These treatments are delivered directly to the lungs, thereby reducing side effects, and may include antibiotics to treat or control infections (e.g. tobramycin), mucolytics to thin sputum making it easier to clear (e.g. dornase alfa), and osmotics to draw water into the sputum making it easier to cough up (e.g. hypertonic saline).

Whilst advances in medicine mean that many of the symptoms of CF can be managed effectively, effective management itself requires people living with $\mathrm{CF}$ to complete a complex daily treatment regimen, comprising daily chest physiotherapy, pancreatic enzymes, nutritional supplements, and inhaled or nebulised therapies. A minimum of one to three nebulised treatments per day are usually prescribed, depending on an individual's characteristics (Hoo 2016), with treatments taking an average of 41 minutes per day (Sawicki 2009).

Treatments are only effective if they are taken, yet evidence suggests that in high-income countries, only $50 \%$ of people with a chronic condition adhere to treatment recommendations (WHO 2003). As with other chronic conditions, low adherence to treatments for CF, including inhaled therapies, is a global problem, with widespread implications for the individual and their family, for CF teams supporting the individual, and for society as a whole. Low adherence to all treatments is associated with an increased number of pulmonary exacerbations requiring intravenous (IV) antibiotics (Eakin 2011) and hospitalisations, which incur higher healthcare costs (Quittner 2014). Two trials have demonstrated the importance of adherence to inhaled therapies on health outcomes, with higher adherence to dornase alfa being associated with a shorter length of hospital stay (Nasr 2013); and individuals collecting four or more courses of nebulised tobramycin per year being $60 \%$ less likely to be admitted to hospital than those collecting two or fewer courses (Briesacher 2011).

Adherence refers to "the extent to which the patient's behaviour matches agreed recommendations from the prescriber" (Horne 2005). This term is more patient-centred and is therefore preferable to the outdated term of compliance, which is "the extent to which the individual's behaviour matches the prescriber's recommendations" (Haynes 1979). There are different methods of measuring adherence, each of which has varying degrees of objectivity and validity. Self-reported measures (e.g. questionnaires and diaries) are inexpensive and easy to administer, but are subject to a range of biases, including social desirability bias and recall bias (Osterberg 2005). Objective count measures (e.g. medication possession ratio (MPR)) address these biases, but they too are prone to overestimation as they do not guarantee the treatment has been taken (Osterberg 2005). Objective recorded measures (e.g. electronic monitoring devices) are a more accurate, albeit expensive, method of measuring adherence (Osterberg 2005). With the development of chipped nebulisers (e.g. Philips I-neb ${ }^{\oplus}$ and PARI eTrack ${ }^{\circledR}$ ) which record time- and date-stamped data, it is possible to measure adherence to inhaled therapies using this method. Trials using objective recorded measures suggest that adherence to inhaled therapies in people with CF is around 31\% to $36 \%$ in adults (Daniels 2011; Hoo 2019; Hoo 2020) and 65\% to $67 \%$ in children or adolescents (Ball 2013; McNamara 2009). The discrepancy between objective and subjective measurement has also been highlighted (Daniels 2011).

\section{Description of the intervention}

Interventions that specifically aim to improve adherence to prescribed medications vary widely (Nieuwlaat 2014). Psychological interventions aimed at improving adherence to inhaled therapies in people with CF can therefore take many forms and may be targeted at: people with CF (e.g. patient education, medication reminders, rewards, psychological therapies); their families (e.g. increasing supervision with medication, family therapy); or the multidisciplinary team (MDT) providing specialist care to the person with CF (e.g. training clinicians to communicate more effectively with patients). Interventions may be delivered face-to-face, over the phone, or using digital technology (e.g. smartphone applications or 'apps' that include rewards, reminders, or providing feedback on treatment-taking behaviour).

Given the complexity of many adherence interventions tested to date (Nieuwlaat 2014), and the way in which complex interventions are reported or described in the literature, it is often difficult to determine the content, or the 'active ingredients', of effective interventions that bring about change (Michie 2009). These 'active ingredients', or behaviour change techniques (BCTs), are defined as the "observable, replicable and irreducible components of an intervention designed to alter or redirect causal processes that regulate behaviour" (Michie 2013). Often, few details are provided about the BCTs used in interventions, and the terminology used to describe interventions is inconsistent. This makes it difficult to replicate effective interventions, because the core components, or 'active ingredients', are not known, and time and resources are wasted because ineffective techniques continue to be implemented (Michie 2009). This review therefore aims to identify the content, or the 'active ingredients', of interventions, in terms of the BCTs used, in order to establish the most effective components of interventions for improving adherence to inhaled therapies in people with CF. This will aid understanding of the causal mechanisms underlying adherence behaviour, which will help inform the design of more effective, consistent and cost-effective interventions in the future (Michie 2009). A reliable, consensuallyagreed taxonomy (BCT Taxonomy version 1; BCTTV1) contains 93 BCTs and provides a common language to identify and describe intervention content (Michie 2013). At least two other reviews have used the BCTTV1 to code the BCTs present in complex interventions (Black 2020; Lawrenson 2016). Psychological interventions for 
improving adherence to inhaled therapies in people with CF may incorporate one or more BCTs (e.g. 'problem-solving', defined as "analysing, or prompting the person to analyse, factors influencing the behaviour and generating or selecting strategies that include overcoming barriers and/or increasing facilitators" (Michie 2013)).

In this review, interventions will be compared to either an active control group or standard care, which usually consists of an annual review or routine reviews (or a combination of both) with a specialist CF MDT.

\section{How the intervention might work}

Reasons for non-adherence are wide-ranging and varied, and understanding these reasons is crucial in order to assist with the development of effective, evidence-based interventions. Previous trials have attempted to understand the factors influencing adherence to treatments in people with CF, and commonly reported reasons include being too busy, forgetting to take a treatment, not having the time to take treatments, and the level of treatment burden (Bregnballe 2011; Dziuban 2010; George 2010; Modi 2006; Sawicki 2015). Treatment beliefs (e.g. lack of perceived benefit, feeling embarrassed about taking treatments in public, believing it is acceptable to miss treatments) have also been reported to influence adherence to treatments in people with CF (Bregnballe 2011; Bucks 2009; Dziuban 2010; George 2010; Sawicki 2015). This is consistent with the Necessity-Concerns Framework (Horne 1999), which posits that beliefs about the necessity of treatments, and concerns about potential adverse consequences, can influence adherence and non-adherence to treatments across a wide range of conditions and medications (Horne 2013).

Whilst treatment beliefs and concerns (i.e. conscious or reflective motivation) may be an important influence on treatment-taking, it is also important to consider the role of automatic motivation influences, with recent trials highlighting the role of habit (i.e. "automatically experiencing an urge to use a nebuliser" (Hoo 2019a)) on adherence to inhaled therapies in adults with CF (Arden 2019; Hoo 2017; Hoo 2019a). Objective adherence data, collected using date- and time- logging nebulisers, have demonstrated that, amongst adolescents with CF, adherence to inhaled therapies is greatest on school days compared to weekends or holidays (Ball 2013), despite this being a time when adolescents are likely to be busiest and are therefore more susceptible to miss treatments due to burden (Hoo 2017).

Given the complexity and multifactorial nature of adherence, interventions aimed at improving adherence to inhaled therapies are likely to be complex, or contain several interacting components (Craig 2008). A 'one size fits all' intervention is therefore unlikely to be effective, as it will need to address issues of capability, opportunity, and motivation (Arden 2019), as highlighted in the COM-B (capability, opportunity, motivation and behaviour) model of behaviour (Michie 2011). Interventions may include reminders or alarms to target forgetting, although evidence for the efficacy of reminders on adherence is limited (Choudhry 2017). Although forgetting is often understood to be 'unintentional' nonadherence, it may be given as a reason to justify 'intentional' non-adherence (Arden 2019; Drabble 2019; George 2010), in a more socially acceptable way (Drabble 2019). Interventions may target the individual's or family members' (e.g. parent) beliefs about the necessity of treatments, and concerns about potential adverse consequences, through psycho-education or problem- solving. They may also focus on supporting people with CF to develop routines and build habits for treatment-taking, e.g. through the use of action planning. Feedback on treatment-taking behaviour may be provided (Demonceau 2013), and interventions may include people with CF self-monitoring their adherence to inhaled therapies, e.g. through the use of chipped nebulisers and digital technology applications. Healthcare professionals also have an important role in supporting adherence to inhaled therapies in adults with CF (Arden 2019). National Institute for Health and Care Excellence guidelines recommend the use of a non-judgemental approach which involves asking open questions and not making assumptions, to promote open and honest conversations in relation to adherence (NICE 2009). Interventions may therefore involve training CF MDT members to communicate more effectively with individuals in relation to adherence, or teaching healthcare professionals how to address necessity beliefs and concerns during consultations (Chapman 2015).

\section{Why it is important to do this review}

A previous Cochrane Review concluded that establishing "effective ways to help people follow medical treatments could have far larger effects on health than any treatment itself" (Haynes 2002). Furthermore, "simplifying the treatment burden" and "improving and sustaining adherence to treatment" have been identified as top $10 \mathrm{CF}$ research priorities in the James Lind Alliance Priority Setting Partnership (Davies 2020; Rowbotham 2017). Adherence research has also been identified as a high priority by the World Health Organisation (WHO 2008). Despite this, there have been no published systematic reviews to assess the effectiveness of psychological interventions for improving adherence to inhaled therapies in people with CF. A previous Cochrane Protocol which focused on adherence to all treatments in CF was withdrawn (Jones 2015). The current Cochrane Review will provide a more detailed focus on CF than general adherence reviews (Nieuwlaat 2014) and will expand upon other $\mathrm{CF}$ reviews focused on self-management interventions (Savage 2014) and psychological interventions for improving a range of outcomes (Goldbeck 2014). Interventions will be coded in order to establish which BCTs are most effective at improving adherence to inhaled therapies in people with CF. By identifying the 'active ingredients' of interventions, the results of this review will help researchers, intervention designers and clinicians better support people with CF. By focusing on inhaled therapies, this review will complement other reviews planned or produced by the group on the theme of adherence (Jones 2020; Smith 2020).

\section{O B JECTIVES}

The primary objective of the review is to assess the efficacy of psychological interventions for improving adherence to inhaled therapies in people with cystic fibrosis (CF). A secondary objective is to establish the most effective components, or behaviour change techniques (BCTs), of interventions for improving adherence to inhaled therapies in people with CF, using the BCT Taxonomy version 1 (Michie 2013). 


\section{METHODS}

\section{Criteria for considering studies for this review}

\section{Types of studies}

We will include randomised controlled trials (RCTs); we will consider quasi-RCTs if we can reasonably assume that the baseline characteristics are similar in both groups. We will include RCTs of parallel design and cluster RCTs; we will only include first-period data from cross-over RCTs due to the likely carry-over effects of the intervention. We will include published and unpublished trials, as well as those published as abstracts only.

\section{Types of participants}

We will include participants of any age, gender, and ethnicity who have a diagnosis of $\mathrm{CF}$ and who are prescribed inhaled therapies (as confirmed in the methodology of each included RCT). We will include participants based in either a hospital or community setting. Trials that include participants with CF and participants with other chronic conditions will only be included if there is a distinction made between the different participant groups (so that non-CF participants with other chronic conditions can be excluded from our analyses). We will also include MDTs or families of people with CF if they are the target participants of an intervention aimed at improving adherence to inhaled therapies in people with CF.

A previous Cochrane Review has highlighted the inclusion bias likely to be present in adherence trials, with many interventions conducted using convenience samples in which participants have higher than average adherence at baseline (Nieuwlaat 2014). This is an important methodological issue to consider as this may reduce the power of an included trial to achieve the primary outcome.

\section{Types of interventions}

We will aim to assess all potential psychological interventions for improving adherence to inhaled therapies in people with CF as detailed above (Description of the intervention) and which are specifically aimed at people with CF, the families of people with CF, or specialist CF MDTs. We will include trials that report at least one measure of adherence as an outcome.

We will use the BCTTv1 (Michie 2013) to identify the BCTs present in interventions in the included trials in order to establish which BCTs are most effective at improving adherence to inhaled therapies in people with CF. We will also code the BCTs present in the active control groups, as the content and effectiveness of these interventions may vary considerably (de Bruin 2016). Given the wide variety of intervention targets included (i.e. children, adolescents and adults with CF, families of people with CF, and specialist (F MDTs), it may not be appropriate to combine the results from different populations in statistical analysis.

\section{Types of outcome measures}

\section{Primary outcomes}

1. Adherence to inhaled therapies (measured using both objective and subjective measures)

a. objective recorded measures (e.g. electronic monitoring devices such as Philips I-neb ${ }^{\circledR}$ and PARI eTrack ${ }^{\circledR}$ nebulisers which record date- and time-stamped data)

b. objective count measures (e.g. MPR)

c. subjective measures (e.g. self-reported adherence; questionnaires; participant diaries)

2. Treatment-related adverse events

a. anxiety as measured by a validated anxiety scale, e.g. GAD-7 (Spitzer 2006)

b. depression as measured by a validated depression scale, e.g. PHQ-8 (Kroenke 2009)

We will include trials with a measure of adherence regardless of if it is a primary or secondary outcome in the trials. If an included trial measures adherence using more than one type of measure, then the most reliable measure will be reported (i.e. objective measures will be preferentially reported over subjective measures, using the hierarchy of validity above). This is consistent with other Cochrane Reviews looking at adherence (Cross 2020; Hollands 2019).

Adherence is expected to be typically defined as a continuous measure (e.g. total number of doses taken as a percentage of the target number of doses prescribed over a given time period). Alternatively, it may be defined as a dichotomous outcome, e.g. reporting whether the treatment is being used to a pre-specified degree (e.g. adherence for $\mathrm{X}$ number of days) or 'adherent' versus 'non-adherent', although this latter approach is overly simplistic and unlikely in our opinion.

Where possible, adherence will refer to 'normative adherence', a measure that takes into account a person's characteristics when defining the minimum required treatment regimen (Hoo 2016). This is in contrast to 'unadjusted adherence', which is where a treatment regimen may be informed by considerations other than treatment effectiveness (e.g. a regimen based on what people with CF feel they can realistically manage rather than a regimen that has been driven by clinical evidence) (Hoo 2016).

\section{Secondary outcomes}

1. Quality of life (QoL) as measured by a validated QoL questionnaire, e.g. CFQ-R (Quittner 2009)

2. Lung function (absolute change or percentage change compared to baseline values or both), measured in forced expiratory volume at one second $\left(\mathrm{FEV}_{1}\right) \mathrm{L}$ or per cent $(\%)$ predicted

3. Pulmonary exacerbations (using the measure specified in the methodology of each trial, e.g. modified Fuchs criteria (Bilton 2011))

a. number of exacerbations

b. time to next exacerbation

c. duration of exacerbations (as measured by the total number of IV days)

If outcomes are reported at multiple time points, we will extract data from all time points. We will group outcome data into those 
measured at up to three months; over three months and up to six months; over six months and up to 12 months; and over 12 months. If trials record outcome data at other time periods, then we will consider examining these as well.

\section{Search methods for identification of studies}

We will search for all relevant published and unpublished trials without restrictions on language, year or publication status. If we identify non-English trials, we will assess these and if necessary, translate these with the assistance of a native speaker (e.g. by posting a request on Cochrane TaskExchange).

\section{Electronic searches}

The Cochrane Cystic Fibrosis and Genetic Disorders Group's Information Specialist will conduct a systematic search of the Group's Cystic Fibrosis Trials Register for relevant trials using the following terms: treatment adherence OR mental health in CF OR behaviour.

The Cystic Fibrosis Trials Register is compiled from electronic searches of the Cochrane Central Register of Controlled Trials (CENTRAL) (updated with each new issue of the Cochrane Library), weekly searches of MEDLINE, a search of Embase to 1995 and the prospective handsearching of two journals - Pediatric Pulmonology and the Journal of Cystic Fibrosis. Unpublished work is identified by searching the abstract books of three major CF conferences: the International Cystic Fibrosis Conference; the European Cystic Fibrosis Conference and the North American Cystic Fibrosis Conference. For full details of all searching activities for the register, please see the relevant section of the Cochrane Cystic Fibrosis and Genetic Disorders Group's website.

The review authors will search the following databases:

- PubMed (www.ncbi.nlm.nih.gov/pubmed/ 1946 to present);

- PsycINFO Ovid (1806 to present);

- Scopus (1823 to present);

- OpenGrey (www.opengrey.eu/).

They will also search the following trials registries:

- World Health Organization International Clinical Trials Registry Platform (WHO ICTRP) (apps.who.int/trialsearch);

- US National Institutes of Health Ongoing Trials Register ClinicalTrials.gov (www.clinicaltrials.gov).

See the appendices for details of the search strategies (Appendix 1).

\section{Searching other resources}

We will check the reference lists of included trials and any relevant systematic reviews identified for further references to relevant trials. We will contact leaders and organisations in the field to obtain additional information on relevant trials.

\section{Data collection and analysis}

\section{Selection of studies}

Two review authors (SD, LC) will independently screen all titles and abstracts identified from the search for their relevance for inclusion in the review based on trial design, types of participants and interventions (as detailed above). We will obtain full-text publications of those deemed either eligible or potentially eligible, as well as for those where eligibility is unclear. We will identify and exclude duplicates and will collate multiple reports of the same trial so that each trial, rather than each report, is the unit of interest in the review. We will independently screen the full-text publications and identify trials for inclusion in the review according to the predetermined eligibility criteria in a prespecified order. We will record the reasons for exclusion of trials in the table 'Characteristics of excluded studies'. The two authors (SD, LC) will resolve any disagreements regarding the inclusion of a trial by discussion or through consultation with a third author if necessary (CG or DCC).

\section{Data extraction and management}

We will develop a data extraction form and pilot this, undertaking any amendments as necessary. We will extract the following main sets of data from each included trial:

- trial details (lead author; date);

- methods (trial design and timetable, randomisation, allocation concealment);

- participants (participant condition(s) and demographics, age, gender, race or ethnicity, socioeconomic status, numbers of participants in each trial arm, trial participant inclusion criteria);

- interventions (content and format of interventions, including details of information provided, intervention setting and delivery provider);

- outcomes (outcome measures, assessment time points, analysis, results);

- additional comments.

Two review authors (SD, LC) will independently extract data and will resolve discrepancies through discussion, or through consultation with a third author if necessary (CG or DCC). Any authors involved in an included trial will not be involved in data extraction for that trial. The lead review author (SD) will enter the data into RevMan, with the second review author (LC) checking the accuracy of the data entry (RevMan 2020).

We will report outcome data at different time points (up to three months; over three months and up to six months; over six months and up to 12 months; and over 12 months). If trial investigators record outcome data at other time periods, then we will consider examining these as well.

We will code the extracted intervention descriptions into component BCTs using the BCTTV1, an established taxonomy of 93 BCTs, as a coding framework (Michie 2013). We will code BCTs for each intended recipient; we will also code each intervention separately, including control arms and will code BCTs as 'present' or 'absent' for each intervention description. As originally described (Michie 2009), and highlighted in a Cochrane Review, complex behaviour change interventions are often poorly reported which makes it challenging to identify the intervention content or BCTs used (Lawrenson 2016). As such, we will contact the authors of included trials about the content of the intervention if there is insufficient information available (Michie 2013). We will request additional materials or information that provides further detail on the content of the intervention (e.g. trial protocol, letters sent to participants). We will code received materials using the taxonomy in the same manner as for the corresponding published reports. Two review authors (SD, LC) will independently conduct BCT 
coding, resolving discrepancies by discussion or by consultation with a third author (CG or DCC) if necessary.

We will narratively record and report any results that we are not able to formally analyse in RevMan (RevMan 2020).

\section{Assessment of risk of bias in included studies}

Two review authors (SD, LC) will independently assess the risk of bias (or methodological quality) for each included trial, with any disagreements resolved by discussion or by consultation with a third author (CG or DCC) if necessary. We will contact the trial authors for additional information about the trial methods if necessary. We will use the Cochrane risk of bias tool to assess the following domains: sequence generation or randomisation; allocation concealment; blinding of participants, personnel and outcomes assessors; incomplete outcome data; and selective outcome reporting (Higgins 2011). However, blinding of participants and personnel is typically not possible in trials of psychological interventions. As such, the assessment of blinding will only include the outcome assessors. We will categorise the overall risk of bias of included trials as low, high, or unclear (according to the criteria specified Higgins 2011), and present this (along with a justification for the judgement) in the tables.

Any authors involved in an included trial will not assess that trial for risk of bias or GRADE.

\section{Measures of treatment effect}

If appropriate (e.g. when interventions, participants and the underlying clinical questions and outcomes are similar enough for pooling to make sense), we will conduct a meta-analysis. When single trials report multiple trial arms, we will only include the relevant arms. For continuous variables, we will calculate mean differences (MDs) with 95\% confidence intervals (Cls); or standardised mean differences (SMDs) with $95 \% \mathrm{Cls}$ when the same outcome is measured on different scales. For dichotomous variables, we will present risk ratios (RRs) with 95\% Cls. For data reported as rates or count data (e.g. number of pulmonary exacerbations), we will calculate rate ratios with $95 \% \mathrm{Cls}$. We expect that most of the data we collect in this review will be continuous given the outcome measures listed (e.g. adherence to inhaled therapies; lung function, $\mathrm{QoL}$ ).

We will narratively describe any skewed data reported as medians and ranges.

\section{Unit of analysis issues}

For cluster RCTs, we will only include data in the meta-analyses if the available data have been adjusted to account for the clustering (or can be adjusted, based on recommendations (Higgins 2019)). For cross-over RCTs, we will include data from the first period only as we cannot exclude a carry-over effect of the intervention.

\section{Dealing with missing data}

If data are missing from the included trials, we will contact the original trial authors (by email, letter or telephone) to obtain these data. If data such as standard deviations (SDs) are missing, where appropriate, we will calculate these from standard errors (SEs), Cls, $\mathrm{t}$-values or $\mathrm{P}$ values (if reported) (Deeks 2019).

\section{Assessment of heterogeneity}

We will assess clinical and methodological heterogeneity by considering the variation in the characteristics of the participants, interventions, outcomes and trial designs. We will visually inspect forest plots and use the $\mathrm{Chi}^{2}$ test and the $\mathrm{I}^{2}$ statistic to measure heterogeneity among the trials included in each analysis. We will consider an $\mathrm{I}^{2}$ value greater than $50 \%$ to represent substantial heterogeneity (Higgins 2011).

\section{Assessment of reporting biases}

We will use a comprehensive search strategy, which includes searching for unpublished trials (grey literature) and searching trials registers (see Search methods for identification of studies) to minimise reporting biases. We will contact the trial authors for the full data or the reason for not publishing the data, if data are not reported or published in full. If we are able to pool more than 10 trials, we will use funnel plots to assess the possibility of publication bias (Page 2019).

\section{Data synthesis}

If trials are sufficiently similar in terms of participants, eligibility criteria, interventions (type and intent) and outcomes (including the timeframe of follow-up and type of measure used), we will consider meta-analysis as described above (Assessment of heterogeneity). We will use a random-effects model and will perform a sensitivity analysis using a fixed-effect model. If we are unable to undertake meta-analyses (e.g. if there are too few included trials or if there is a substantial level of heterogeneity), we will present a narrative synthesis of the included trials.

\section{Subgroup analysis and investigation of heterogeneity}

If sufficient data are available, we will conduct subgroup analyses with respect to:

1. the target intervention participants (e.g. children and adolescents with CF; adults with CF; families of people with CF; or MDTs). In trials that include participants with a range of ages (i.e. children and adolescents or adults or unspecified), we will classify adults with CF as 18 years and over and children and adolescents with CF as under 18 years of age; and

2. the BCTs used.

If appropriate, we will investigate the impact of type and number of BCTs on effect size by meta-regression.

\section{Sensitivity analysis}

If we can include a sufficient number of trials in any meta-analysis, we will undertake sensitivity analyses to assess the robustness of the results by excluding trials with an unclear or high risk of overall bias. This may include excluding trials in which adherence was measured using subjective methods (e.g. self-reported adherence; questionnaires; participant diaries).

\section{Summary of findings table}

We will develop a summary of findings table for each comparison using the following outcomes at the 'over six months and up to 12 months' time point: 
1. adherence to inhaled therapies (\% completed treatments) (reported using SMD for all results combined from six to 12 months);

2. change from baseline in anxiety (as measured by a validated anxiety scale, e.g. GAD-7 (Spitzer 2006));

3. change from baseline in depression (as measured by a validated depression scale, e.g. PHQ-8 (Kroenke 2009));

4. change from baseline in $\mathrm{QoL}$ (as measured by a validated $\mathrm{QoL}$ questionnaire, e.g. CFQ-R (Quittner 2009));

5. change from baseline in $\mathrm{FEV}_{1} \%$ predicted;

6. number of pulmonary exacerbations, as measured using the measure specified in the methodology of each trial, e.g. modified Fuchs criteria (Bilton 2011).
We will generate a table for each comparison that is presented in the review (e.g. intervention versus standard care; and intervention versus active control group).

We will use the five GRADE considerations (risk of bias, consistency of effect, imprecision, indirectness and publication bias) to assess the certainty of the body of evidence for each outcome, and to draw conclusions about the certainty of evidence in the review (Schünemann 2019).

\section{ACKNOWLEDGEMENTS}

This project was supported by the National Institute for Health Research, via Cochrane Infrastructure funding to the Cochrane Cystic Fibrosis and Genetic Disorders Group. The views and opinions expressed therein are those of the authors and do not necessarily reflect those of the Systematic Reviews Programme, NIHR, NHS or the Department of Health. 


\section{RE F E R E N C E S}

\section{Additional references}

\section{Arden 2019}

Arden MA, Drabble S, O'Cathain A, Hutchings M, Wildman M. Adherence to medication in adults with Cystic Fibrosis: An investigation using objective adherence data and the Theoretical Domains Framework. British Journal of Health Psychology 2019;24(2):357-380. [DOI: 10.1111/bjhp.12357]

\section{Ball 2013}

Ball R, Southern KW, McCormack P, Duff AJA, Brownlee KG, McNamara PS. Adherence to nebulised therapies in adolescents with cystic fibrosis is best on week-days during school termtime. Journal of Cystic Fibrosis 2013;12(5):440-4. [DOI: 10.1016/ j.jcf.2012.12.012]

\section{Bell 2020}

Bell SC, Mall MA, Gutierrez H, Macek M, Madge S, Davies JC, et al. The future of cystic fibrosis care: a global perspective. The Lancet Respiratory Medicine Commission 2020;8:65-124. [DOI: 10.1016/S2213-2600(19)30337-6]

\section{Bilton 2011}

Bilton D, Canny G, Conway S, Dumcius S, Hjelte L, Proesmans M, et al. Pulmonary exacerbation: towards a definition for use in clinical trials. Report from the EuroCareCF Working Group on outcome parameters in clinical trials. Journal of Cystic Fibrosis 2011;10(2):S79-81. [DOI: 10.1016/S1569-1993(11)60012-X]

\section{Black 2020}

Black N, Johnston M, Michie S, Hartmann-Boyce J, West R, Viechtbauer W, et al. Behaviour change techniques associated with smoking cessation in intervention and comparator groups of randomized controlled trials: a systematic review and metaregression. Addiction 2020 March 20 [Epub ahead of print]. [DOI: 10.1111/add.15056]

\section{Bregnballe 2011}

Bregnballe V, Schiøtz PO, Boisen KA, Pressler T, Thastum M. Barriers to adherence in adolescents and young adults with cystic fibrosis: a questionnaire study in young patients and their parents. Patient Preference and Adherence 2011;5:507-515. [DOI: 10.2147/PPA.S25308]

\section{Briesacher 2011}

Briesacher BA, Quittner AL, Saiman L, Sacco P, Fouayzi H, Quittell LM. Adherence with tobramycin inhaled solution and health care utilization. BMC Pulmonary Medicine 2011;11(5). [DOI: 10.1186/1471-2466-11-5]

\section{Bucks 2009}

Bucks RS, Hawkins K, Skinner TC, Horn S, Seddon P, Horne R. Adherence to treatment in adolescents with cystic fibrosis: The role of illness perceptions and treatment beliefs. Journal of Pediatric Psychology 2009;34(8):893-902. [DOI: 10.1093/jpepsy/ jsn135]

\section{CF Foundation Patient Registry 2019}

CF Foundation Patient Registry. 2018 Annual Data Report. https://www.cff.org/Research/Researcher-Resources/PatientRegistry/2018-Patient-Registry-Annual-Data-Report.pdf (accessed 23 August 2020).

\section{Chapman 2015}

Chapman SCE, Barnes N, Barnes M, Wilkinson A, Hartley J, Piddock $C$, et al. Changing adherence-related beliefs about ICS maintenance treatment for asthma: feasibility study of an intervention delivered by asthma nurse specialists. BMJ Open 2015;5:e007354. [DOI: 10.1136/bmjopen-2014-007354]

\section{Choudhry 2017}

Choudhry NK, Krumme AA, Ercole PM, Girdish C, Tong AY, Khan NF, et al. Effect of reminder devices on medication adherence: the REMIND randomized clinical trial. JAMA Internal Medicine 2017;177(5):624-631. [DOI: 10.1001/ jamainternmed.2016.9627]

\section{Craig 2008}

Craig P, Dieppe P, Macintyre S, Michie S, Nazareth I, Petticrew M. Developing and evaluating complex interventions: the new Medical Research Council guidance. BMJ 2008;337:a1655. [DOI: 10.1136/bmj.a1655]

\section{Cross 2020}

Cross AJ, Elliott RA, Petrie K, Kuruvilla L, George J. Interventions for improving medication-taking ability and adherence in older adults prescribed multiple medications. Cochrane Database of Systematic Reviews 2020, Issue 5. Art. No: CD012419. [DOI: 10.1002/14651858.CD012419.pub2]

\section{Cystic Fibrosis Canada 2019}

Cystic Fibrosis Canada. The Canadian Cystic Fibrosis Registry 2018 Annual Data Report. Available from https://www.cysticfibrosis.ca/uploads/ RegistryReport2018/2018RegistryAnnualDataReport.pdf 2019.

\section{Cystic Fibrosis Trust 2018}

Cystic Fibrosis Trust. What is cystic fibrosis? www.cysticfibrosis.org.uk/what-is-cystic-fibrosis (accessed 24 October 2019).

\section{Daniels 2011}

Daniels T, Goodacre L, Sutton C, Pollard K, Conway S, Peckham D. Accurate assessment of adherence: self-report and clinician report vs electronic monitoring of nebulizers. Chest 2011;140(2):425-32. [DOI: 10.1378/chest.09-3074]

\section{Davies 2020}

Davies G, Rowbotham NJ, Smith S, Elliot ZC, Gathercole K, Rayner $\mathrm{O}$, et al. Characterising burden of treatment in cystic fibrosis to identify priority areas for clinical trials. Journal of Cystic Fibrosis 2020;19:499-502. [DOI: 10.1016/j.jcf.2019.10.025]

\section{de Bruin 2016}

de Bruin M, Viechtbauer W, Eisma MC, Hartmann-Boyce J, West R, Bull E, et al. Identifying effective behavioural 
components of Intervention and Comparison group support provided in SMOKing CEssation (IC-SMOKE) interventions: a systematic review protocol. Systematic Reviews 2016;5(77). [DOI: 10.1186/s13643-016-0253-1]

\section{Deeks 2019}

Deeks JJ, Higgins JP, Altman DG (editors). Chapter 10: Analysing data and undertaking meta-analyses. In: Higgins JP, Thomas J, Chandler J, Cumpston M, Li T, Page MJ, Welch VA (editors). Cochrane Handbook for Systematic Reviews of Interventions version 6.0 (updated July 2019). Cochrane, 2019. Available from www.training.cochrane.org/handbook.

\section{Demonceau 2013}

Demonceau J, Ruppar T, Kristanto P, Hughes DA, Fargher E, Kardas $\mathrm{P}$, et al. Identification and assessment of adherenceenhancing interventions in studies assessing medication adherence through electronically compiled drug dosing histories: A systematic literature review and meta-analysis. Drugs 2013;73:545-562. [DOI: 10.1007/s40265-013-0041-3]

\section{Drabble 2019}

Drabble SJ, O'Cathain A, Arden MA, Hutchings M, Beever D, Wildman $M$. When is forgetting not forgetting? A discursive analysis of differences in forgetting talk between adults with cystic fibrosis with different levels of adherence to nebulizer treatments. Qualitative Health Research 2019;29(14):2119-31. [DOI: 10.1177/1049732319856580]

\section{Dziuban 2010}

Dziuban EJ, Saab-Abazeed L, Chaudhry SR, Streetman DS, Nasr SZ. Identifying barriers to treatment adherence and related attitudinal patterns in adolescents with cystic fibrosis. Pediatric Pulmonology 2010;45:450-458. [DOI: 10.1002/ ppul.21195]

\section{Eakin 2011}

Eakin MN, Bilderback A, Boyle MP, Mogayzel PJ, Riekert KA. Longitudinal association between medication adherence and lung health in people with cystic fibrosis. Journal of Cystic Fibrosis 2011;10(4):258-64. [DOI: 10.1016/j.jcf.2011.03.005]

\section{ECFS Patient Registry 2017}

Zolin A, Orenti A, Naehrlich L, van Rens J, Fox A, Krasnyk M, et al. European Cystic Fibrosis Society Patient Registry Annual Data Report 2017. www.ecfs.eu/sites/default/files/generalcontent-images/working-groups/ecfs-patient-registry/ ECFSPR_Report2017_v1.3.pdf (accessed 24 October 2019).

\section{George 2010}

George M, Rand-Giovannetti D, Eakin MN, Borrelli B, Zettler M, Riekert KA. Perceptions of barriers and facilitators: Selfmanagement decisions by older adolescents and adults with CF. Journal of Cystic Fibrosis 2010;9:425-432. [DOI: 10.1016/ j.jcf.2010.08.016]

\section{Goldbeck 2014}

Goldbeck L, Fidika A, Herle M, Quittner AL. Psychological interventions for individuals with cystic fibrosis and their families. Cochrane Database of Systematic Reviews 2014, Issue 6. Art. No: CD003148. [DOI: 10.1002/14651858.CD003148.pub3]

\section{Haynes 1979}

Haynes RB, Taylor DW, Sackett DL. Compliance in health care. Baltimore: Johns Hopkins University Press, 1979.

\section{Haynes 2002}

Haynes R, McDonald HP, Garg A, Montague P. Interventions for helping patients to follow prescriptions for medications. Cochrane Database of Systematic Reviews 2002, Issue 2. Art. No: CD000011. [DOI: 10.1002/14651858.CD000011]

\section{Higgins 2011}

Higgins JP Altman DG, Sterne JA on behalf of the Cochrane Statistical Methods Group and the Cochrane Bias Methods Group, editor(s). Chapter 8: Assessing risk of bias in included studies. In: Higgins JP, Green S, editor(s). Cochrane Handbook for Systematic Reviews of Interventions. Version 5.1.0 (updated March 2011). The Cochrane Collaboration, 2011. Available from handbook.cochrane.org.

\section{Higgins 2019}

Higgins JP, Eldridge S, Li T (editors). Chapter 23: Including variants on randomized trials. In: Higgins JP, Thomas J, Chandler J, Cumpston M, Li T, Page MJ, Welch VA (editors). Cochrane Handbook for Systematic Reviews of Interventions version 6.0 (updated July 2019). Cochrane, 2019. Available from www.training.cochrane.org/handbook.

\section{Hollands 2019}

Hollands GJ, Naughton F, Farley A, Lindson N, Aveyard P. Interventions to increase adherence to medications for tobacco dependence. Cochrane Database of Systematic Reviews 2019, Issue 8. Art. No: CD009164. [DOI: 10.1002/14651858.CD009164.pub3]

\section{Hoo 2016}

Hoo ZH, Curley R, Campbell MJ, Walters SJ, Hind D, Wildman MJ. Accurate reporting of adherence to inhaled therapies in adults with cystic fibrosis: methods to calculate "normative adherence". Patient Preference and Adherence 2016;10:887-900. [DOI: 10.2147/PPA.S105530]

\section{Hoo 2017}

Hoo ZH, Boote J, Wildman MJ, Campbell MJ, Gardner B. Determinants of objective adherence to nebulised medications among adults with cystic fibrosis: an exploratory mixed methods study comparing low and high adherers. Health Psychology and Behavioral Medicine 2017;5:299-316. [DOI: 10.1080/21642850.2017.1338958]

\section{Hoo 2019}

Hoo ZH, Totton N, Waterhouse S, Hind D, Girling C, Bradburn M, et al. Understanding objective adherence to preventative inhaled therapies at a centre level for quality improvement - a CFHealthHub (CFHH) improvement collaborative study. Journal of Cystic Fibrosis 2019;18:S51-2. [DOI: 10.1016/ S1569-1993(19)30283-8]

\section{Hoo 2019a}

Hoo ZH, Gardner B, Arden MA, Waterhouse S, Walters SJ, Campbell MJ, et al. Role of habit in treatment adherence among 
adults with cystic fibrosis. Thorax 2019;74(2):197-199. [DOI: 10.1136/thoraxjnl-2017-211453]

\section{Hoo 2020}

Hoo ZH, Curley R, Walters SJ, Campbell MJ, Wildman MJ. Exploring the implications of different approaches to estimate centre-level adherence using objective adherence data in an adult cystic fibrosis centre - a retrospective observational study. Journal of Cystic Fibrosis 2020;19(1):162-7. [DOI: 10.1016/ j.jcf.2019.10.008]

\section{Horne 1999}

Horne R, Weinman J. Patients' beliefs about prescribed medicines and their role in adherence to treatment in chronic physical illness. Journal of Psychosomatic Research 1999;47(6):555-567. [DOI: 10.1016/S0022-3999(99)00057-4]

\section{Horne 2005}

Horne R, Weinman J, Barber N, Elliott R, Morgan M, Cribb A, et al. Concordance, adherence and compliance in medicine taking: report for the National Co-ordinating Centre for NHS Service Delivery and Organisation R\&D (NCCSDO). www.netscc.ac.uk/ hsdr/files/project/SDO_FR_08-1412-076_V01.pdf (accessed 24 October 2019).

\section{Horne 2013}

Horne R, Chapman SCE, Parham R, Freemantle N, Forbes A, Cooper V. Understanding patients' adherence-related beliefs about medicines prescribed for long-term conditions: A metaanalytic review of the Necessity-Concerns Framework. PLoS One 2013;8(12):e80633. [DOI: 10.1371/journal.pone.0080633]

\section{Jones 2015}

Jones S, Curley R, Wildman M, Morton RW, Elphick HE. Interventions for improving adherence to treatment in cystic fibrosis. Cochrane Database of Systematic Reviews 2018, Issue 7. Art. No: CD011665. [DOI: 10.1002/14651858.CD011665.pub2]

\section{Jones 2020}

Jones M, Moffatt F, Harvey A, Ryan JM. Interventions for improving adherence to airway clearance treatment and exercise in people with cystic fibrosis. Cochrane Database of Systematic Reviews 2020, Issue 5. Art. No: CD013610. [DOI: 10.1002/14651858.CD013610]

\section{Kroenke 2009}

Kroenke K, Strine TW, Spritzer RL, Williams JB, Berry JT, Mokdad AH. The PHQ-8 as a measure of current depression in the general population. Journal of Affective Disorders 2009;114(1-3):163-73. [DOI: 10.1016/j.jad.2008.06.026]

\section{Lawrenson 2016}

Lawrenson JG, Graham-Rowe E, Lorencatto F, Presseau J, Burr J, Ivers N, et al. Interventions to increase attendance for diabetic retinopathy screening. Cochrane Database of Systematic Reviews 2016, Issue 1. Art. No: CD012054. [DOI: 10.1002/14651858.CD012054]

\section{McNamara 2009}

McNamara PS, McCormack P, McDonald AJ, Heaf L, Southern KW. Open adherence monitoring using routine data download from an adaptive aerosol delivery nebuliser in children with cystic fibrosis. Journal of Cystic Fibrosis 2009;8(4):258-63. [DOI: 10.1016/j.jcf.2009.04.006]

\section{Michie 2009}

Michie S, Fixsen D, Grimshaw JM, Eccles MP. Specifying and reporting complex behaviour change interventions: the need for a scientific method. Implementation Science 2009;4(40). [DOI: 10.1186/1748-5908-4-40]

\section{Michie 2011}

Michie S, van Stralen MM, West R. The behaviour change wheel: a new method for characterising and designing behaviour change interventions. Implentation Science 2011;6(42). [DOI: 10.1186/1748-5908-6-42]

\section{Michie 2013}

Michie S, Richardson M, Johnston M, Abraham C, Francis J, Hardeman W, et al. The behavior change technique taxonomy (v1) of 93 hierarchically clustered techniques: building an international consensus for the reporting of behavior change interventions. Annals of Behavioral Medicine 2013;46(1):81-95. [DOI: 10.1007/s12160-013-9486-6]

\section{Modi 2006}

Modi AC, Quittner AL. Barriers to treatment adherence for children with cystic fibrosis and asthma: what gets in the way? Journal of Pediatric Psychology 2006;31(8):846-858. [DOI: 10.1093/jpepsy/jsj096]

\section{Nasr 2013}

Nasr SZ, Chou W, Villa KF, Chang E, Broder MS. Adherence to dornase alfa treatment among commercially insured patients with cystic fibrosis. Journal of Medical Economics 2013;16(6):1-8. [DOI: 10.3111/13696998.2013.787427]

\section{NICE 2009}

National Institute for Health and Care Excellence (NICE). Medicines adherence: involving patients in decisions about prescribed medicines and supporting adherence. Available from https://www.nice.org.uk/guidance/cg76 2009.

\section{Nieuwlaat 2014}

Nieuwlaat R, Wilczynski N, Navarro T, Hobson N, Jeffery R, Keepanasseril A, et al. Interventions for enhancing medication adherence. Cochrane Database of Systematic Reviews 2014, Issue 11. Art. No: CD000011. [DOI: 10.1002/14651858.CD000011.pub4]

\section{Osterberg 2005}

Osterberg L, Blaschke T. Adherence to medication. New England Journal of Medicine 2005;353(5):487-97. [DOI: 10.1056/ NEJMra050100]

\section{Page 2019}

Page MJ, Higgins JP, Sterne JA. Chapter 13: Assessing risk of bias due to missing results in a synthesis. In: Higgins JP, Thomas J, Chandler J, Cumpston M, Li T, Page MJ, Welch VA (editors). Cochrane Handbook for Systematic Reviews of Interventions version 6.0 (updated July 2019). Cochrane, 2019. Available from www.training.cochrane.org/handbook. 


\section{Quittner 2009}

Quittner AL, Modi AC, Wainwright C, Otto K, Kirihara J, Montgomery AB. Determination of the minimal clinically important difference scores for the Cystic Fibrosis Questionnaire-Revised respiratory symptom scale in two populations of patients with cystic fibrosis and chronic Pseudomonas aeruginosa airway infection. Chest 2009;135(6):1610-8.

\section{Quittner 2014}

Quittner AL, Zhang J, Marynchenko M, Chopra PA, Signorovitch J, Yushkina Y, et al. Pulmonary medication adherence and health-care use in cystic fibrosis. Chest 2014;146(1):142-51. [DOI: 10.1378/chest.13-1926]

\section{RevMan 2020 [Computer program]}

The Cochrane Collaboration Review Manager 5 (RevMan 5). Version 5.4. The Cochrane Collaboration, 2020.

\section{Rowbotham 2017}

Rowbotham NJ, Smith S, Leighton PA, Rayner OC, Gathercole K, Elliott ZC, et al. The top 10 research priorities in cystic fibrosis developed by a partnership between people with CF and healthcare providers. Thorax 2018;73(4):388-90. [DOI: 10.1136/ thoraxjnl-2017-210473]

\section{Savage 2014}

Savage E, Beirne PV, Ni Chroinin M, Duff A, Fitzgerald T, Farrell D. Self-management education for cystic fibrosis. Cochrane Database of Systematic Reviews 2014, Issue 9. Art. No: CD007641. [DOI: 10.1002/14651858.CD007641.pub3]

\section{Sawicki 2009}

Sawicki GS, Sellers DE, Robinson WM. High treatment burden in adults with cystic fibrosis: Challenges to disease selfmanagement. Journal of Cystic Fibrosis 2009;8:91-96. [DOI: 10.1016/j.jcf.2008.09.007]

\section{Sawicki 2015}

Sawicki GS, Heller KS, Demars N, Robinson WM. Motivating adherence among adolescents with cystic fibrosis: youth and parent perspectives. Pediatric Pulmonology 2015;50:127-136. [DOI: 10.1002/ppul.23017]

\section{Schünemann 2019}

Schünemann HJ, Higgins JP, Vist GE, Glasziou P, Akl EA, Skoetz N, et al (editors). Chapter 14: Completing 'Summary of findings' tables and grading the certainty of the evidence. In: Higgins JP, Thomas J, Chandler J, Cumpston M, Li T, Page MJ,
Welch VA (editors). Cochrane Handbook for Systematic Reviews of Interventions version 6.0 (updated July 2019). Cochrane, 2019. Available from www.training.cochrane.org/handbook.

\section{Smith 2018}

Smith S, Rowbotham NJ, Regan KH. Inhaled anti-pseudomonal antibiotics for long-term therapy in cystic fibrosis. Cochrane Database of Systematic Reviews 2018, Issue 3. Art. No: CD001021. [DOI: 10.1002/14651858.CD001021.pub3]

\section{Smith 2020}

Smith S, Calthorpe R, Herbert S, Smyth AR. Digital technology for monitoring adherence to inhaled therapies in people with cystic fibrosis. Cochrane Database of Systematic Reviews 2020, Issue 9. Art. No: CD013733. [DOI: 10.1002/14651858.CD013733]

\section{Spitzer 2006}

Spitzer RL, Kroenke K, Williams JBW, Löwe B. A brief measure for assessing generalized anxiety disorder: the GAD-7. Archives of Internal Medicine 2006;166(10):1092-7. [DOI: 10.1001/ archinte.166.10.1092]

\section{UK CF Registry 2018}

UK Cystic Fibrosis Trust. UK Cystic Fibrosis Registry: annual data report 2018. www.cysticfibrosis.org.uk/ /media/documents/ the-work-we-do/uk-cf-registry/2018-registry-annual-datareport.ashx?la=en (accessed 24 October 2019).

\section{UK CF Registry 2019}

UK Cystic Fibrosis Trust. UK Cystic Fibrosis Registry: annual data report 2019. www.cysticfibrosis.org.uk/ /media/documents/ the-work-we-do/uk-cf-registry/registry-report-2019.ashx?la=en (accessed 23 August 2020).

\section{WHO 2003}

World Health Organisation. Adherence to long-term therapies: evidence for action. whqlibdoc.who.int/ publications/2003/9241545992.pdf (accessed 24 October 2019).

\section{WHO 2008}

World Health Organisation. Global Priorities for Research in Patient Safety (first edition). www.who.int/patientsafety/research/priorities/ global_priorities_patient_safety_research.pdf (accessed 24 October 2019).

\section{Yang 2018}

Yang C, Montgomery M. Dornase alfa for cystic fibrosis. Cochrane Database of Systematic Reviews 2018, Issue 9. Art. No: CD001127. [DOI: 10.1002/14651858.CD001127.pub4]

\section{APPENDICES}

\section{Appendix 1. Search Methods - Electronic Searches}

\begin{tabular}{ll}
\hline Database/Resource & Strategy \\
\hline PubMed & $\# 1$ randomized controlled trial [pt
\end{tabular}


(Continued)

(1946 to present)

\author{
\#2 controlled clinical trial [pt] \\ \#3 randomized [tiab] \\ \#4 placebo [tiab] \\ \#5 drug therapy [sh] \\ \#6 randomly [tiab] \\ \#7 trial [tiab]
}

\#8 groups [tiab]

\#9 \#1 OR \#2 OR \#3 OR \#4 OR \#5 OR \#6 OR \#7 OR \#8

\#10 animals [mh] NOT humans [mh]

\#11 \#9 NOT \#10

\#12 cystic fibrosis"[MeSH Terms] OR "cystic fibrosis" OR mucoviscidosis OR mucoviscidose

\#13 adhere OR adherence OR adhered OR nonadherence OR persist OR persistance OR persisted OR compliance OR comply OR complied OR noncompliance OR concordance OR non concordance OR cooperative OR cooperation OR cooperate OR cooperated OR uncooperative OR conform

\#14 "psychology"[MeSH Terms] OR "mind-body therapies"[MeSH Terms] OR "psychotherapy"[MeSH Terms] OR "psychology, applied"[MeSH Terms] OR "neuropsychology"[MeSH Terms] OR Psycholog* OR Psychosocial OR psychotherapy OR psychotherapies OR psychotherapist OR psychoanal $^{*}$ OR psychodrama OR behavio* OR attitude* OR communicat* OR reward* OR remind* OR educate OR education OR supervis ${ }^{\star}$ OR family* OR families OR train ${ }^{\star}$ OR monitor ${ }^{\star}$ OR problem ${ }^{\star}$ OR team ${ }^{\star}$ OR diary OR diaries OR counsel ${ }^{\star}$ OR feedback OR reinforc ${ }^{\star}$ OR motivat ${ }^{\star}$ OR self-regulat* OR cope OR coping OR emotion* OR social ${ }^{\star}$ OR sociol ${ }^{\star}$ OR cognitive OR cognition OR mental ${ }^{\star}$ OR verbal* OR construct OR crisis OR aversion OR assertiv* OR accept ${ }^{\star}$ OR mind OR mindful* OR mood* OR support* OR wellness OR habit* OR talk* OR depress* OR goal* OR support* OR hypnosis OR hypnotherap*

\title{
\#15 \#11 AND \#12 AND \#13 AND \#14
}

*NOTE: Lines \#1- \#11 are the Cochrane Highly Sensitive Search Strategy for identifying randomized trials in MEDLINE: sensitivity-maximizing version (2008 revision); PubMed format

\section{PsychINFO Ovid}

Search terms to be based on PubMed search strategy

(1806 to present)

Scopus

(1823 to present)
1 cystic fibrosis OR mucoviscidosis OR mucoviscidose [Article title, Abstract, Keywords]

2 adhere OR adherence OR adhered OR nonadherence OR persist OR persistance OR persisted OR compliance OR comply OR complied OR noncompliance OR concordance OR non concordance OR cooperative OR cooperation OR cooperate OR cooperated OR uncooperative OR conform

3 Psycho* OR behavio $^{\star}$ OR attitude* OR communicat ${ }^{\star}$ OR reward ${ }^{\star}$ OR remind ${ }^{\star}$ OR educat ${ }^{\star}$ OR supervis $^{\star}$ OR famil ${ }^{\star}$ OR train* OR monitor ${ }^{\star}$ OR problem ${ }^{\star}$ OR team ${ }^{\star}$ OR diar ${ }^{\star}$ OR counsel ${ }^{\star}$ OR feedback OR reinforc ${ }^{\star}$ OR motivat* ${ }^{\star}$ OR self-regulat ${ }^{\star}$ OR cope OR coping OR emotion*

4 social $^{\star}$ OR socio* OR cognitive OR mental* OR verbal* OR condition* OR knowledge OR personal OR construct OR crisis OR aversion OR assertiv* OR Accept* OR commit ${ }^{\star}$ OR mind* OR mood* OR support $^{\star}$ OR well ${ }^{\star}$ OR habit* OR talk* OR depress*

\section{5 \#3 OR \#4}

\section{6 \#1 AND \#2 AND \#5}


*NOTE: maximum number of characters exceeded in Line \#3, so psychological search terms divided into two search lines and combined using OR operator (Line \#5)

\begin{tabular}{ll}
\hline OpenGrey & (cystic fibrosis OR mucoviscidosis OR mucoviscidose) AND (adhere OR adherence OR adhered OR \\
& nonadherence OR persist OR persistance OR persisted OR compliance OR comply OR complied OR \\
& noncompliance OR concordance OR non concordance OR cooperative OR cooperation OR cooper- \\
ate OR cooperated OR uncooperative OR conform)
\end{tabular}

[Advanced Search]
CONDITION/ DISEASE: cystic fibrosis OR mucoviscidosis OR mucoviscidose
OTHER TERMS: adhere OR adherence OR adhered OR nonadherence OR persist OR persistance OR
persisted OR compliance OR comply OR complied OR noncompliance OR concordance OR non con-
cordance OR cooperative OR cooperation OR cooperate OR cooperated OR uncooperative OR con-
form
STUDY TYPE: Interventional Studies (Clinical Trials)

WHO ICTRP Search terms to be based on Clinicaltrials.gov search strategy

\section{HISTORY}

Protocol first published: Issue 10, 2020

\section{CONTRIBUTIONS OF AUTHORS}

\begin{tabular}{ll}
\hline Task & Author(s) responsible \\
\hline Protocol stage: draft the protocol & SD; LC \\
\hline Review stage: select which trials to include (2+1 arbiter) & SD; LC; CG; DCC \\
\hline Review stage: extract data from trials (2 people) & SD; LC \\
\hline Review stage: enter data into RevMan & SD \\
\hline Review stage: carry out the analysis & SD; LC; DCC \\
\hline Review stage: interpret the analysis & SD; LC; DCC \\
\hline Review stage: draft the final review & SD; LC; CG; DCC \\
\hline Update stage: update the review & SD \\
\hline
\end{tabular}

\section{DECLARATIONS OF INTEREST}

SD is employed by Nottingham University Hospitals NHS Trust to work on the CFHealthHub Data Observatory, which aims to embed objective adherence data into routine CF care using quality improvement cycles. She has not had any involvement in the CFHealthHub RCT which took place at 19 CF Centres across the UK to evaluate a complex behaviour change intervention to support adherence to inhaled therapies in people with CF.

LC declares no potential conflict of interest.

DCC declares no potential conflict of interest. 
CG is employed by the University of Sheffield, Clinical Trials Research Unit to manage a clinical trial related to supporting people with CF with adherence behaviours "CFHealthHub Data Observatory" (IRAS 216782 portfolio 33331); the funding is from NHS England.

\section{SOURCES OF SUPPORT}

\section{Internal sources}

- No sources of support supplied

\section{External sources}

- National Institute for Health Research, UK

This systematic review was supported by the National Institute for Health Research, via Cochrane Infrastructure funding to the Cochrane Cystic Fibrosis and Genetic Disorders Group. 